This department welcomes short notes and problems believed to be new. Contributors should include solutions where known, or background material to the problem in case the problem is unsolved. Send all communications concerning this department to Leo Moser, University of Alberta, Edmonton, Alberta.

\title{
ODD AND EVEN PERMUTATIONS
}

\author{
Israel Halperin \\ (received April 1, 1960)
}

This note gives a proof for the familiar elementary theorem that if a permutation of the integers $1, \ldots, n$ (with $\mathrm{n} \geqslant 2$ ) is expressed as a product $\pi_{1}$ of $\mathrm{N}_{1}$ transpositions and also as a product $\pi_{2}$ of $N_{2}$ transpositions, then $N_{1}$ and $N_{2}$ are both even or both odd (equivalently: $\mathrm{N}_{1}+\mathrm{N}_{2}$ is even).

Here, a transposition means an interchange of two of the integers. If the interchange is between adjacent integers it is called an adjacent-trans position.

Since an arbitrary transposition can be expressed as the product of an odd number of adjacent-transpositions (as is easily seen), the theorem need only be proved for the case that $\pi_{1}$ and $\pi_{2}$ are products of adjacent-transpositions. For this case, the theorem can be deduced easily from the following lemma.

LEMMA. If $\mathrm{P}$, the product of $\mathrm{N}$ adjacent-transpositions on $(1, \ldots, n)$, is the identity permutation, then $N$ is even.

Indeed, $\pi_{1}$ followed by the inverse of $\pi_{2}$ gives the identity permutation and the lemma yields: $N=N_{1}+N_{2}$ is even.

As for the lemma itself it holds trivially when $\mathrm{n}=2$; and it can be proved for $\mathrm{n}>2$ by induction, as follows. 
1) The members of $P$ which displace the integer $n$ are even in number because the algebraic sum of the displacements of $n$ by members of $P$ must be 0 .

2) The members of $P$ which displace the integer $n$ are precisely those which do not change the relative order of the other integers $1,2, \ldots, n-1$. Hence if the integer $n$ is ignored the other members of $P$ are adjacent-transpositions on $(1, \ldots, n-1)$ with a product which is the identity permutation. By the inductive assumption, their number must be even.

Thus $N$ is the sum of two even integers and is therefore even.

Queen's University

A LEMMA ON CONTINUOUS FUNCTIONS

\author{
J. Lipman \\ (received December 7, 1959)
}

The point of this note is to get a lemma which is useful in treating homotopy between paths in a topological space [1].

As explained in the reference, two paths joining a given pair of points in a space $E$ are homotopic if there exists a mapping $F: I \times I \rightarrow E$ (I being the closed interval $[0,1]$ ) which deforms one path continuously into the other. In practice, when two paths are homotopic and the mapping $F$ is constructed, then the verification of all its required properties, with the possible exception of continuity, is trivial. The snag occurs when $F$ is a combination of two or three functions on different subsets of $I \times I$. Then the boundary lines between these subsets have to be given special consideration, and although the problems resulting are routine their disposal can involve some tedious calculation and repetition. In the development [1] of the fundamental group of a space, for example, this sort of situation comes up four or five times.

The calculation can be by-passed by means of the 